\title{
CHARGE INHOMOGENEITY AND HIGH TEMPERATURE SUPERCONDUCTIVITY
}

\author{
V.J. EMERY \\ Dept. of Physics, Brookhaven National Laboratory, Upton, NY 11973-5000, USA \\ S.A. KIVELSON \\ Dept. of Physics, UCLA, Los Angeles, CA 90095, USA
}

\begin{abstract}
When the condensation of a gas of fermions into a (self-bound) liquid state is frustrated by the long-range Coulomb interaction, the consequence is a large local fluctuation of the charge density, together with pairing on a high energy scale. The competition between these two effects at long length scales determines the nature of the ordered state at low temperatures. Evidence for the central role of this competition in determining the physical properties of the high temperature superconductors is provided by the delicate interplay of superconductivity, charge and spin ordering, and structural phase transformations in the $\mathrm{La}_{2} \mathrm{CuO}_{4}$ family of materials. There the gas-liquid transition corresponds to the phase separation of holes doped into an antiferromagnetic insulator. Because of the low superfluid density and poor conductivity, the critical temperature for the superconducting transition in underdoped and optimally doped materials is governed by the onset of phase coherence and not by the pairing energy scale.
\end{abstract}

\section{Introduction}

The fundamental driving force for high temperature superconductivity and for many of the unusual properties of the cuprates is the frustration of the motion of holes in an antiferromagnet. Neutral holes are unstable to phase separation into an antiferromagnetically ordered insulating region and a hole-rich metallic region ${ }^{1,2}$. This is the best compromise between the hole kinetic energy and the exchange interaction between the spins. Since superexchange is itself a kinetic process, phase separation minimizes the total zero-point energy of the strongly-correlated system. For charged holes the long-range Coulomb interaction frustrates phase separation, unless the dopants are mobile enough to counterbalance the effect of the long-range Coulomb interaction, as in the case of photodoping or oxygen doping ${ }^{3,4}$. Of course this does not mean that phase separation is without consequence. Between the spinodals, the instability of the neutral system (which is signalled by a negative compressibility) is converted by the Coulomb interaction into charge inhomogeneity on intermediate time scales or length scales, and especially into ordered or fluctuating charge stripes ${ }^{5,6}$. As we shall see, the other consequence is local pairing on a high energy scale. Typically, charge ordering and superconducting phase coherence compete at long length scales, although they may coexist in specific regions of the phase diagram ${ }^{7}$.

There is extensive experimental evidence to show that: 1) Phase separation is the fate of neutral holes in an antiferromagnet ${ }^{3,4}$. 2) The $\mathrm{La}_{2} \mathrm{CuO}_{4}$ family displays ordered ${ }^{8}$ or fluctuating ${ }^{9,10,11}$ charge stripes together with antiphase spin domains. 3) Charge order and superconductivity compete with each other ${ }^{12,13,14}$.

The existence of charge-density wave fluctuations adds a new dimension to discussions of the physics of the high temperature superconductors which, almost from the outset, have been dominated by the interplay of antiferromagnetism and superconductivity. The clearest 
evidence for this behavior is to be found in the $\mathrm{La}_{2} \mathrm{CuO}_{4}$ family although, as we shall see, angle resolved photoemission data on other materials may also be understood from this point of view. Nevertheless the three phenomena are simply different manifestations of a common underlying physical theme, and the best way to investigate any one of them is to find the material in which it shows up the most clearly.

The charge stripes are topological defects, which form antiphase domain walls in the antiferromagnetic order of the background spins ${ }^{8}$. We have argued ${ }^{15}$ that this kind of "topological doping" is a common feature of correlated insulators such as polyacetylene (for which the defects are solitons) and the high temperature superconductors, and that it is responsible for the rapid restoration of the symmetry of the ground state upon doping; non-topological defects would produce much more modest reduction in the magnitude of the order parameter, proportional to the dopant concentration.

\section{Evidence for Stripes.}

Recent neutron scattering studies ${ }^{8}$ of $\mathrm{La}_{1.6-x} \mathrm{Nd}_{0.4} \mathrm{Sr}_{x} \mathrm{CuO}_{4}$ with $x=0.12$ found a succession of transitions; first to the low temperature tetragonal (LTT) structure, then to a charged-ordered state (charge-stripes) and finally, at a slightly lower temperature, to a period-doubling magnetically-ordered state. We conclude that the transitions are driven by frustrated kinetic phase separation because the charge order appears first and the relatively low charge density along the stripes (one doped hole per two $\mathrm{Cu}$ ions) allows the holes to be mobile. The alternative route to stripe order, (a Hartree-Fock, Fermi surface instability ${ }^{16}$ ) predicts a single transition to a charge and spin ordered insulating state, with one doped hole per $\mathrm{Cu}$, twice as large as observed.

$\mathrm{La}_{1.6-x} \mathrm{Nd}_{0.4} \mathrm{Sr}_{x} \mathrm{CuO}_{4}$ with $x=0.12$ does not exhibit bulk superconductivity. But there are substantial low-energy stripe fluctuations in optimally-doped, superconducting samples of $\mathrm{La}_{2-x} \mathrm{Sr}_{x} \mathrm{CuO}_{4}$; inelastic neutron scattering experiments ${ }^{9,10,11}$ have found peaks at energies as low as a few $\mathrm{meV}$ and in essentially the same positions in $k$-space as in (ordered) $\mathrm{La}_{1.6-x} \mathrm{Nd}_{0.4} \mathrm{Sr}_{x} \mathrm{CuO}_{4}$. Clearly the stripe correlations are an essential part of the physics of these materials.

The single-particle properties of a disordered stripe phase ${ }^{17}$ also account for the peculiar features of the electronic structure of high temperature superconductors observed by angle-resolved photoemission spectroscopy (ARPES) on hole-doped high temperature superconductors. In particular, the spectral function of holes moving in a disordered stripe background reproduces the experimentally-observed shape of the Fermi surface and the existence of nearly dispersionless states in its neighborhood. The essential ingredient in this interpretation of the data is a background of slowly-fluctuating stripes whose dynamics is determined by collective effects (the competition between phase separation and the long-range Coulomb force), rather than the single-particle behavior. 


\section{The Mechanism}

As might be expected, an appreciation of the electronic structure and the underlying physics also leads to a natural mechanism of high temperature superconductivity. When the holes on the stripes tunnel into the intervening region, which has a low density of doped holes and strong antiferromagnetic correlations, they experience the very same effective attraction that would have caused them to coalesce (phase separate) in the absence of the longer range Coulomb interaction. In fact, the holes moving on a stripe form a quasi one-dimensional electron gas in an "active" environment which has low-energy excitations in the spin (and possibly charge) degrees of freedom. The environment renormalizes the kinetic energy of the holes and mediates effective interactions between them ${ }^{18}$. We have found several such processes that lead to pairing, even though the basic Hamiltonian contains only repusive interactions ${ }^{18}$. In particular, when two holes hop into the antiferromagnetic environment, they may form a bound state, which typically has a large binding energy and a low mobility, and cannot, by itself, lead to high temperature superconductivity. However, the holes in the stripe are able to combine this large binding energy with their own mobility and achieve a high superconducting transition temperature ${ }^{18}$.

\section{Phase Fluctuations}

It is possible to define a characteristic temperature for superconducting phase ordering $T_{\theta}^{m a x}$ which is proportional to the phase stiffness $V_{0}=(\hbar c)^{2} d / 16 \pi e^{2} \lambda^{2}(0)$ where $\lambda(0)$ is the zero-temperature penetration depth and $d$ is a the spacing between the $\mathrm{CuO}_{2}$ planes. We have shown ${ }^{19}$ that, if $T_{c} \ll T_{\theta}^{\max }$, then phase fluctuations are relatively unimportant and $T_{c}$ is likely to be close to the mean-field transition temperature $T^{M F}$ predicted by BCS theory. On the other hand, if $T_{\theta}^{\max } \sim \mathcal{T}_{c}$, then the value of $\mathcal{T}_{c}$ is determined primarily by phase ordering, and $T^{M F}$ is simply the characteristic temperature below which local pairing becomes significant.

The value of $T_{\theta}^{\max } / T_{c}$ for the cuprate superconductors is of order unity ${ }^{19}$, which places them in a quite different category from conventional superconductors, for which the ratio can be as large as $10^{5}$. Indeed, $T_{c}$ is predominantly determined by phase fluctuations in underdoped high temperature superconductors and by the mean-field transition temperature $T^{M F}$ in overdoped materials such as $\mathrm{Tl} 2201\left(T_{\theta}^{\max } / T_{c} \geq 2\right)$. Optimally doped materials, such as $\mathrm{YBa}_{2} \mathrm{Cu}_{3} \mathrm{O}_{7-\delta}$ and $\mathrm{La}_{2-x} \mathrm{Sr}_{x} \mathrm{CuO}_{4}$, with $\delta$ and $x$ in the neighborhood of 0.05 and 0.15 respectively, are in the crossover region between the two.

When $T_{c}$ is much smaller than $T^{M F}$, the existence of pairing without phase coherence should be manifested as a pseudogap in the the temperature range $T_{c}<T<T^{M F}$. Such a pseudogap has been observed in underdoped samples of high temperature superconductors, such as $\mathrm{YBa}_{2} \mathrm{Cu}_{3} \mathrm{O}_{7-\delta}$ and $\mathrm{Bi}_{2} \mathrm{Sr}_{2} \mathrm{CaCu}_{2} \mathrm{O}_{8+x}$ and in the stoichiometric material $\mathrm{YBa}_{2} \mathrm{Cu}_{4} \mathrm{O}_{8}$. The pseudogap has been seen in spin ${ }^{20}$, charge ${ }^{21,22,23}$, and single-electron ${ }^{24}$ properties. Moreover, the central peak in the $a$-axis optical conductivity (which becomes a $\delta$-function in the superconducting state) already narrows above $\mathrm{T}_{c}{ }^{25}$ in $\mathrm{YBa}_{2} \mathrm{Cu}_{4} \mathrm{O}_{8}$ and underdoped $\mathrm{YBa}_{2} \mathrm{Cu}_{3} \mathrm{O}_{7-\delta}$. The prediction ${ }^{19}$ that $\mathrm{T}_{c}$ is supressed by phase fluctuations in organic conductors has been confirmed by the observation ${ }^{26}$ of pseudogap behavior in 
the nuclear spin relaxation rate. Taken as a whole, these experiments strongly support the existence of a phase fluctuation regime in the cuprate and organic superconductors.

Acknowledgements: We have benefitted from discussions with John Tranquada on all aspects of this problem and especially on charge ordering in the nickelates and cuprates. This work was supported in part by NSF grant \#DMR-93-12606 and by the Division of Materials Sciences, U.S. Department of Energy, under contract DE-AC02-76CH00016.

\section{References}

1. V.J. Emery and S.A. Kivelson, and H-Q Lin, Phys. Rev. Eett. 64, 475-478 (1990).

2. S.A. Kivelson and V.J. Emery, in Strongly Correlated Electronic Materials: The Los Alamos Symposium 1993, ed. by K.S. Bedell, et al. (Addison Wesley, Redwood City, 1994) p. 619.

3. V.J. Emery and S.A. Kivelson, Physica C 209594 (1993).

4. Phase Separation in Cuprate Superconductors, eds. K. A. Müller and G. Benedek (World Scientfic Singapore, 1993); Proc. of the Second and Third International Confs. on Phase Separation in Cuprate Superconductors, Cottbus, Germany, 1993 and Erice, Sicily, July 1995.

5. U. Löw et al. Phys. Rev. Lett. 72, 1918 (1994).

6. L. Chayes et al., Physica $\mathbf{A}$, in press.

7. V.J. Emery and S.A. Kivelson, to be published.

8. J. Tranquada et al., Nature 375, 561 (1995) and to be published.

9. S-W. Cheong, et al., Phys. Rev. Lett. 67, 1791-1794 (1991).

10. T.E. Mason, et al., Phys. Rev. Lett. 68, 1414-1417 (1992).

11. T. R. Thurston, et al., Phys. Rev. B 46, 9128-9131 (1992).

12. B. Büchner it et al., Phys. Rev. Lett. 73, 1841 (1994).

13. M. Nohara it et al., Phys. Rev. Lett. 70, 3447 (1993).

14. C-H. Lee it et al., Sendai preprint.

15. V.J. Emery and S.A. Kivelson, Sissa preprint cond-mat/9603009, to be published in Synthetic Metals.

16. J. Zaanen and O. Gunnarsson, Phys. Rev. B 40, 7391 (1989); papers cited in ref. 2.

17. M.I. Salkola, V.J. Emery and S.A. Kivelson, Proc. of the Third International Conf. on Phase Separation in High $T_{c}$ Superconductors, Erice, July, 1995 (in press) and submitted to Physical Review Letters.

18. V.J. Emery and S.A. Kivelson, O. Zachar, to be published.

19. V.J. Emery and S.A. Kivelson, Nature 374, 434 (1995).

20. M. Mehring, Appl. Magn. Reson. 3, 383-421 (1992).

21. D.N. Basov, et al., Phys. Rev. B 50, 3511-3514 (1994).

22. P. Wachter, B. Bucher, and R. Pittini, Phys. Rev. B 49, 13164-13171 (1994).

23. D.N. Basov, et al., Phys. Rev. Lett. 74, 598-601 (1995).

24. D. S. Marshall et al., (Stanford University Preprint) submitted to Phys. Rev. Lett..

25. D.N. Basov et al., McMaster University preprint.

26. H. Mayaffre et al., Europhys. Lett., 28, 205 (1994). 


\section{DISCLAIMER}

This report was prepared as an account of work sponsored by an agency of the United States Government. Neither the United States Government nor any agency thereof, nor any of their employees, makes any warranty, express or implied, or assumes any legal liability or responsibility for the accuracy, completeness, or usefulness of any information, apparatus, product, or process disclosed, or represents that its use would not infringe privately owned rights. Reference herein to any specific commercial product, process, or service by trade name, trademark, manufacturer, or otherwise does not necessarily constitute or imply its endorsement, recommendation, or favoring by the United States Government or any agency thereof. The views and opinions of authors expressed herein do not necessarily state or reflect those of the United States Government or any agency thereof. 
